\title{
Fibrosis Deep Connective Tissue, CTCAE
}

National Cancer Institute

\section{Source}

National Cancer Institute. Fibrosis Deep Connective Tissue, CTCAE. NCI Thesaurus. Code C143487.

A disorder characterized by fibrotic degeneration of the deep connective tissues. 